\title{
A Qualitative Study Exploring Factors Associated with Provider Adherence to Severe Pediatric Traumatic Brain Injury Guidelines
}

\author{
Sarah M. Brolliar, ${ }^{1}$ Megan Moore, Hilaire J. Thompson, ${ }^{1}$ Lauren K. Whiteside, ${ }^{1}$ Richard B. Mink, ${ }^{2}$ \\ Mark S. Wainwright, ${ }^{3}$ Jonathan I. Groner, ${ }^{4}$ Michael J. Bell, ${ }^{5}$ Christopher C. Giza, ${ }^{6}$ Douglas F. Zatzick, \\ Richard G. Ellenbogen, Linda Ng Boyle, Pamela H. Mitchell, Frederick P. Rivara, ${ }^{1}$ and Monica S. Vavilala ${ }^{1}$
}

\begin{abstract}
Despite demonstrated improvement in patient outcomes with use of the Pediatric Traumatic Brain Injury (TBI) Guidelines (Guidelines), there are differential rates of adherence. Provider perspectives on barriers and facilitators to adherence have not been elucidated. This study aimed to identify and explore in depth the provider perspective on factors associated with adherence to the Guidelines using 19 focus groups with nurses and physicians who provided acute management for pediatric patients with TBI at five university-affiliated Level 1 trauma centers. Data were examined using deductive and inductive content analysis. Results indicated that three inter-related domains were associated with clinical adherence: 1) perceived guideline credibility and applicability to individual patients, 2) implementation, dissemination, and enforcement strategies, and 3) provider culture, communication styles, and attitudes towards protocols. Specifically, Guideline usefulness was determined by the perceived relevance to the individual patient given age, injury etiology, and severity and the strength of the evidence. Institutional methods to formally endorse, codify, and implement the Guidelines into the local culture were important. Providers wanted local protocols developed using interdisciplinary consensus. Finally, a culture of collaboration, including consistent, respectful communication and interdisciplinary cooperation, facilitated adherence. Provider training and experience, as well as attitudes towards other standardized care protocols, mirror the use and attitudes towards the Guidelines. Adherence was determined by the interaction of each of these guideline, institutional, and provider factors acting in concert. Incorporating provider perspectives on barriers and facilitators to adherence into hospital and team protocols is an important step toward improving adherence and ultimately patient outcomes.
\end{abstract}

Key words: brain injury; evidence-based medicine; guideline adherence; injury; pediatrics; trauma

\section{Introduction}

T RAUMATIC BRAIN INJURY (TBI) persists as a leading cause of morbidity and mortality for children ages 0 to 19 years of age. ${ }^{1}$ Acute TBI treatment is complex, involving interdisciplinary care and ancillary services across the emergency department, operating room, and critical care unit. In an effort to improve outcomes, the Pediatric TBI Guidelines $^{2}$ (Guidelines) were developed to create a standard for optimal treatment and represent a compilation of evidence-based recommendations for the medical management of children younger than 18 years of age with severe TBI as defined by a Glasgow Coma Scale (GCS) score of $3-8 .^{2}$ The initial Guide- lines ${ }^{2}$ provided the foundation for provision of acute care in this population, though neither those nor the 2012 revision $^{3}$ addressed implementation or adherence strategies. Pediatric TBI Guideline adherence during the first $72 \mathrm{~h}$ after hospital admission has been associated with better survival and discharge outcomes as measured by the Glasgow Outcome Scale (GOS). ${ }^{4,5}$ Therefore, it is important to understand provider and organizational factors that lead to improved use and adoption of these guidelines at Level 1 pediatric trauma centers, which in turn will lead to improved patient outcomes.

The goal of this study was to identify the facilitators and potentially remediable barriers to severe Pediatric TBI Guideline ${ }^{2,3}$

\footnotetext{
${ }^{1}$ Harborview Injury Prevention and Research Center, University of Washington, Seattle, Washington.

${ }^{2}$ Harbor-University of California; Los Angeles BioMedical Research Institute, Los Angeles, California.

${ }^{3}$ Ann and Robert H. Lurie Children's Hospital of Chicago, Chicago, Illinois.

${ }^{4}$ Ohio State University College of Medicine, Columbus, Ohio.

${ }^{5}$ University of Pittsburgh, Pittsburgh, Pennsylvania.

${ }^{6}$ Mattel Children's Hospital, University of California, Los Angeles, Los Angeles, California.
} 
adherence from the provider perspective. We focused on the experiences of providers at Level 1 trauma centers because of the high volume of severe pediatric TBI patients treated at these centers. ${ }^{6}$

\section{Methods}

\section{Study centers}

We conducted focus groups at five geographically dispersed Level 1 pediatric trauma centers in the continental United States that treat 30-50 children with severe TBI annually and were affiliated with universities. We identified cases with TBI between June 2012 and May 2013. These facilities were approached for collaboration as part of the Pediatric Guideline Adherence and Outcomes (PEGASUS) study as sites represented in the Pediatric Neurocritical Care Research Group (www.pncrg.org). These sites were the most likely to care for children with severe TBI in their region. Study sites were the University of Washington, Seattle, Washington (lead and data coordinating center); Children's Hospital of Pittsburgh, Pittsburgh, Pennsylvania; Ann and Robert H. Lurie Children's Hospital of Chicago, Chicago, Illinois; Harbor-University of California, Los Angeles Medical Center, Torrance, CA; and Nationwide Children's Hospital, Columbus, Ohio. Two investigators (MSV and MJB) were on the committee that developed the Guidelines. The study was granted a designation of minimal risk (expedited review) and a waiver of written documentation of consent by the University of Washington. Each study site's human subjects' committee approved the protocol and the University of Washington coordinated all study efforts.

\section{Participants}

Physicians and nurses (e.g., providers) who provided acute treatment for severe pediatric TBI patients were purposively recruited at all sites. We framed the inclusion criteria in this way in order to recruit providers identified with a variety of subspecialties. At each of the participating sites, participants included providers in the intensive care unit and emergency department. Representation of the following subspecialties were included in the groups: emergency medicine, trauma, neurosurgery, critical care, pediatrics, and anesthesiology. Participation was voluntary, confidential, and uncompensated. Recruitment of additional focus groups continued until we reached saturation, when no new themes emerged. ${ }^{7}$ See Table 1 for details about the number of physician and nurse focus groups at each site.

\section{Interviews}

Participants were given a copy of the 2012 Pediatric TBI Guidelines $^{3}$ for reference prior to the discussion. Focus groups were facilitated by trained and experienced moderators. All PEGASUS

Table 1. Physician and Nurse Focus Groups by Site

\begin{tabular}{lcc}
\hline & $\begin{array}{c}\text { MD focus } \\
\text { groups }\end{array}$ & $\begin{array}{c}\text { RN focus } \\
\text { groups }\end{array}$ \\
\hline $\begin{array}{l}\text { Lurie Children's Hospital of Chicago, } \\
\quad \text { Illinois }\end{array}$ & 2 & 1 \\
$\begin{array}{l}\text { Nationwide Children's Hospital- } \\
\quad \text { Columbus, Ohio }\end{array}$ & 1 & 1 \\
$\begin{array}{l}\text { University of Washington } \\
\text { Harbor-University of California, } \\
\quad \text { Los Angeles }\end{array}$ & 4 & 6 \\
$\begin{array}{l}\text { Children's Hospital of Pittsburg, } \\
\quad \text { Pennsylvania }\end{array}$ & 1 & 1 \\
Total & $\mathbf{9}$ & 1 \\
\hline
\end{tabular}

MD, physician; RN, nurse. investigators participated in the development of the discussion guide. The guide included open-ended questions aimed at eliciting perspectives on facilitators and barriers to successful adoption of the Guidelines, as well as organizational factors and local characteristics that impacted adherence to the Guidelines and potential solutions to problems identified.

Separate focus groups were held for physicians and nurses to minimize hierarchical conflicts and increase the reliability of the data. ${ }^{8}$ No identifying information was gathered. Interviews were facilitated in person on the respective hospital campus at the University of Washington or via video conferencing by the same two moderators. The average duration of each focus group was 32 min (range, 18$49 \mathrm{~min}$ ). Focus groups were audio recorded and transcribed verbatim. Participants were assigned a number to substitute for the use of names, allowing differentiation in the transcript while preserving anonymity.

\section{Analysis}

Two investigators read and independently coded the transcribed interviews using NVivo 9.0 (QSR International Pty, Doncaster, Victoria, Australia) qualitative data analysis software to capture and organize thematic units. Patterns were identified in the data using content analysis. ${ }^{9}$ Both deductive and inductive content analysis were used, the former utilizing a priori hypotheses based on theory, the latter to derive themes directly from the data for the creation of a codebook. ${ }^{10}$ Focus groups were the unit of analysis. Codes were retained for the final codebook if they met a threshold number of references (at least 20) and distribution across focus groups (at least six groups), or were novel to severe pediatric TBI. Exceptional cases were incorporated to ensure the model was credible and comprehensive. ${ }^{11}$ On average, codes appeared in 12.5 of the 19 total focus groups. Investigators used the codebook and a selection of transcripts for context to iteratively sort codes and create categories under five a priori and inductively determined domains: environment/institution, communication, staffing, guideline attributes, and individual factors. ${ }^{9}$ Using the a priori domains as scaffolding, we condensed and arranged redundant categories to explore potential mechanisms and relationships between them. Discrepancies were discussed among the team members through a series of in-person meetings until consensus was reached on the preliminary model. The preliminary model was compared again to the data and finalized with input from the larger research group. Data interpretation was enhanced by the presence of an expert interdisciplinary team. ${ }^{9}$ Models of thematic domains are presented in Figure 1 and illustrative quotes are presented in Table 2.

\section{Results}

\section{Study sample}

We conducted nine focus groups with physicians and 10 focus groups with nurses across five hospital sites. A total of 54 physicians and 74 nurses participated. Participation ranged between two and eight physicians and three and 12 nurses per focus group. General disclosure of a provider's specialty within the transcript confirmed at minimum the presence of emergency medicine physicians; general, trauma, and neurosurgeons; intensive care, critical care, and pediatric critical care physicians; anesthesiologists; residents, fellows, and attending physicians; and emergency department, operating room, and intensive care unit/pediatric intensive care unit nurses. Two study sites treated both adult and pediatric patients.

\section{Findings}

We created a model of factors related to adherence. Barriers and facilitators of adherence to the Pediatric TBI Guidelines ${ }^{2,3}$ were identified and encompass three domains and associated factors: 


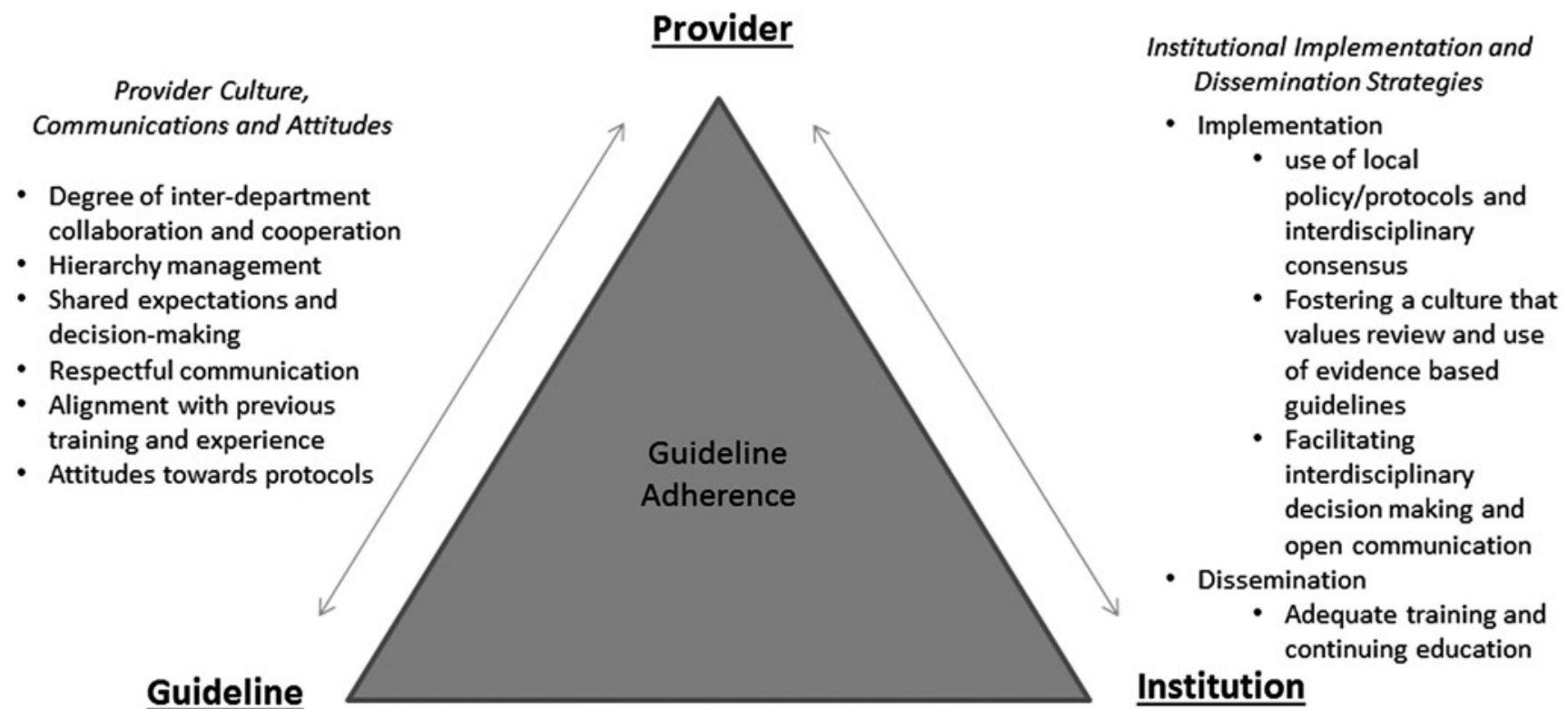

Guideline Credibility and Applicability

- Guideline Credibility: perceived strength of the evidence

- Guideline Applicability: perceived relevance to the individual patient/injury

FIG. 1. Triangle of pediatric traumatic brain injury adherence.
1) guideline credibility and applicability to individual patients, 2) implementation, dissemination, and enforcement strategies at the institution, and 3) provider culture, communication and attitudes towards protocols. A model of domains and themes is illustrated in Figure 1.

Domain 1: Guideline credibility and applicability. Adherence to the Guidelines was based on physician belief that the Guidelines were relevant to the patient and their specific injury. This belief appeared to be especially strong in cases of non-accidental trauma or intentional injury, poor prognosis, young patient age, and the presence of multisystem trauma. Many physicians observed or provided differential intracranial pressure (ICP) monitoring and cranial decompression based on whether the TBI was accidental or intentional, believing intentional injury etiology resulted in TBI diagnoses that were inappropriate to Guideline-recommended treatment or the prognosis to be too poor. Similar patterns emerged for infant ICP monitoring and placement of external ventricular drains; some physicians did not believe the benefit of placement in infants outweighed the risks. See Table 1 for illustrative quote. Nurses disagreed about whether treatment differences existed for non-accidental trauma patients and infants. Nurses reported often initiating conversations with physicians to advocate early ordering of nutrition and other TBI care protocols. Some nurses reported using discretion as to when to advocate based on patient characteristics, such as if they believed the child was too ill to benefit from nutrition. Other nurses were initially not aware of the Guideline recommendations for nutrition, and so did not advocate for these orders. Once they became aware, they reported they would advocate for following this recommendation.
Physicians at facilities that care for both adult and pediatric patients discussed the lack of adequate supporting evidence as a limiting credibility factor, and reported the practice of applying the adult TBI guidelines ${ }^{12}$ to children. The reputability of the Pediatric TBI Guideline ${ }^{3}$ authors also influenced credibility. See Table 2 for illustrative quotes.

Domain 2: Implementation and dissemination at the institutional level. Participants reported that institutions can support Guideline adherence by using a formal process for integrating evidence-based guidelines into clinical practice, specifically by incorporating provider involvement and preferences into the local implementation and dissemination strategy (e.g., through an interdisciplinary trauma council). This included determining which parts of the Guidelines to follow, and how adherence would be defined and measured in treatment plans. Standardized treatment protocols based on the Guidelines codified an institution's endorsement; local precedent, cultural norms, and enforcement of protocol use impacted adherence. Providers favored protocols that were developed and implemented using consensus models across all relevant services. Nurses emphasized the need for consensus by citing confusion and inconsistency when working with attending physicians who each had their own preferred treatment plan. One hospital strategy was to hold a joint annual review of the internal TBI guidelines with critical care, neurology, and neurosurgery based on published criteria to support local adherence. At a minimum, physicians and nurses wanted to be apprised of the hospital's policy.

Physicians said that academic publication of the Guidelines was insufficient to raise awareness, and nurse focus groups revealed 
Table 2. Factors Related to Pediatric TBi Guideline Adherence

Theme

Illustrative quotes

\section{Guideline credibility and applicability}

Guidelines' relevance to the "They (the Guidelines) don't specifically say they're applicable to them or not...some people patient and injury might try to use the same criteria for placement of an ICP monitor in a non-accidental trauma patient whereas it might be totally different that the nature of the injury there, a big black brain, can be a different entity, plus they have open fontanels, it's a different patient population." [Physician]

Strength of the evidence

"We probably never [will] have class I evidence... we're going to have to use what we've got." [Physician]

Implementation and dissemination at institution

Implementation

"It would be helpful if we decided as a group what guidelines to follow." [Nurse]

"Local institutional discussion or at least acknowledgement of where the guidelines are going to fall in local practice practice...one could say a local clinical pathway or protocol (is needed)." [Physician]

"The guidelines are posted at the bed side. It's not absolute protocol to go over every parameter, but often we will." [Nurse]

Dissemination and continuing education

“Anywhere you look you're going to see our protocol...we all know exactly the parameters we want." [Nurse]

"Dissemination of information is key every time a new pediatric team comes on every month." [Physician]

Provider culture, communication, and attitudes

Communication/hierarchy management

"Our nurses are empowered to pick up the phone and call the fellows, call the attending, and they don't hesitate to advocate for the patients. It's not a certain hierarchy that we have to go through physicians. [Nurse]

"The hardest part...is two different services who have different philosophies on how to take care of patients, and who often don't communicate. We're left to be the person who is communicating, and that makes for delayed care." [Nurse]

Decision-making culture

"We have a very close teamwork relationship, and so there's no barrier in picking up the phone and saying, things are not going well, we need additional evaluation, we need surgical intervention." [Physician]

"The number one barrier appears to be a surgeon who insists or thinks that...their input or their ideas are more important than whatever consensus data or steps should be." [Physician]

Coordination

"I think we work together as a team by effective patient hand-off to make sure that all the guidelines are communicated effectively and around. We go over, all the team members are able to ask questions and provide input. [Nurse]

"Having a separate service and having a point man...to bridge the gap between critical care and our neurosurgical colleagues has really facilitated a lot of placements of ICP monitors in the past year.' [Physician]

Attitudes toward protocols

"There have to be appropriate avenues for deviation or alteration for individual patients." [Physician]

"We don't treat numbers here, we look at the whole picture...the [protocol] takes the thinking out of it." [Nurse] And I think that undermines what we do here." [Nurse]

Alignment with providers' clinical experience and training

"The surgeons want to apply adult guidelines to pediatric patients... where there is no evidence, no data whatsoever in children, but that's what they do in adults now." [Physician]

"I trained under a very rigid model of TBI care...it was normal saline for 72 hours with nothing else...I've done it one way so many times. Like trying to break an old habit. [Physician]

TBI, traumatic brain injury; ICP, intracranial pressure.

differences in dissemination between facilities. Both physicians and nurses recommended Guideline inclusion in the resident curriculum and hospital- based continuing education to refresh relevant clinical skills and knowledge. Both groups advocated posting the Guidelines for nurses to establish common goals and support communication, and for physicians to settle treatment disputes. Nurses also requested standard treatment codes and bundles, including pre-checked boxes on standing orders for pediatric TBI upon admission. See Table 2 for illustrative quotes.

Domain 3: Provider culture, communication, and attitudes. Individual and team behaviors and communication styles were frequently cited as barriers or facilitators to Guideline adherence, as they relate to interdisciplinary decision-making and care coordination. How providers on different services communicated with each other was critical to maintaining continuity of care and a shared understanding of the treatment plan.

Respectful communication between professionals and across departments facilitated cooperation, namely recognition of interprofessional experience, prompt response to another's concerns, and the absence of hierarchical barriers to communication. Respect also was demonstrated by one's ability to seek a second opinion and an attitude of collaboration. Employing departmental liaisons circumvented poor communication and the lack of trust inherent in 
clinical rotators, such as resident physicians, by having an experienced, permanent person interface with decision makers. Assigning blame, failing to notify co-managing services or to respond to requests, and preventing another from appealing to a second opinion created discord. Overall, teams functioned best when a culture of collaboration was present. This was reflected in communication styles that showed respect for the experience of others, listening across disciplines and the ability to be flexible in the decision-making process.

Guideline adherence became more complex and created unique communication challenges when separate departments were responsible for jointly managing patients. Co-managing departments benefitted from consistent, shared expectations of the decisionmaking process and notification of changes in patient status. Without those expectations, tensions arose when, for instance, in an effort to save time, intensive care unit providers did not solicit opinions of subspecialty services.

Training, experience, and general attitudes towards standardized protocols influenced adherence to the Guidelines. Specifically, adherence was influenced by normative use of protocols locally among providers. Many physicians and nurses reported that a uniform treatment protocol was beneficial for setting common expectations and grounding for discussion, but did not eliminate either disagreement among specialists or dissent in things such as fever and seizure management when physicians deviated in favor of their own clinical opinion. Most nurses and some physicians practicing in hospitals without protocols were dissatisfied with the inconsistency of current care, as it varied according to attending physician preferences. These nurses advocated standing orders for severe pediatric TBI admissions. Resistant physicians desired latitude to exercise their clinical expertise and depart from the Guidelines. Strong Guideline alignment with a physician's clinical experience and post-graduate medical education training were facilitators of adherence. Physicians were more likely to follow recommendations consistent with their residency or fellowship instruction, even when they were aware of the clinical benefits of altering their routine and adhering to the Guidelines. ${ }^{3}$ A minority of nurses and physicians viewed protocols as reductionist and undermining of their experience. Providers were more likely to adhere to protocols that aligned with their training and experience and had built in avenues for provider-driven deviation. See Table 2 for illustrative quotes.

\section{Discussion}

This study used semi-structured focus groups of physicians and nurses across a variety of pediatric trauma centers to explore barriers and facilitators of severe Pediatric TBI Guideline ${ }^{3}$ adherence. Three domains emerged as important to adherence: 1) guideline credibility and applicability to individual patients; 2) implementation, dissemination, and enforcement strategies at the institution; and 3) provider culture, communication, and attitudes towards protocols. The model we present (Fig. 1) illustrates that adherence is determined by the interaction of guideline, institutional, and provider factors acting in concert. ${ }^{13}$ We build upon existing knowledge related to guideline implementation and adherence strategies in critical care. ${ }^{14-16}$ Previous studies have shown that physicians' and nurses' decisions to use evidence-based clinical practice guidelines are broadly influenced by their knowledge and attitudes about guidelines, local professional norms, and institutional factors. ${ }^{16-22}$ Unique to this study is the examination of the interdisciplinary dynamics and unique needs associated with acute management of pediatric TBI.
Our findings support previous studies that have shown adherence is enhanced when providers are sufficiently skilled in the content and have confidence in the recommendation's ability to produce the desired outcome. ${ }^{18-20,22}$ In our study, participants reported that Guideline applicability to a particular patient was determined by the physician, but nurses influenced adherence by differentially advocating for protocol initiation. The distinguishing factors used to decide whether or not to follow the Guidelines included: patient age, injury etiology (intentional vs. accidental trauma), and TBI diagnosis. Physicians' stated rationale for treating intentional trauma patients differently (because of poor prognoses, infancy, or atypical TBI diagnoses) may suggest systematic bias against applying the Guidelines in cases of abusive trauma. Prior studies suggest patients with intentional injury or non-accidental trauma may present differently, compared with patients with similar injuries from a non-intentional etiology. Specifically, they have lower initial GCS scores, ${ }^{23}$ different clinical TBI diagnosis (e.g., type of hemorrhage, location of skull fractures), ${ }^{3-25}$ and ultimately increased morbidity and mortality. ${ }^{26}$ However Guideline ${ }^{3}$ adherence is associated with better outcomes for both groups. ${ }^{4}$ Future revisions may wish to consider the inclusion of abusive head trauma as within the Guideline's purview.

Providers at hospitals that care for both adult and pediatric trauma patients discussed the use of the adult TBI guidelines ${ }^{12}$ on children and the limitations of the evidence supporting the Pediatric TBI Guidelines ${ }^{3}$ as a factor in their analysis of Guideline credibility. This suggests additional barriers to adherence at these facilities or for their providers. However, the study by Vavilala and colleagues showed similar Pediatric TBI Guideline adherence scores between pediatric trauma centers and hospitals with pediatric and adult trauma center status. ${ }^{4}$ Future research is needed to understand how this differentiation impacts care for pediatric severe TBI. We recommend institutional oversight to ensure that the Pediatric TBI Guidelines are applied to all pediatric patients.

Previous studies have shown that overcoming personal and institutional inertia, possessing adequate time, training, and resources, and agreement and communication among the clinical providers were adherence factors for both physicians and nurses. ${ }^{16,18-22}$ In our study, physicians and nurses often spoke about the role of institutions in promoting Guideline use. Hospitals promoted adherence by leveraging policy to codify, enact, and enforce the use of the Guidelines into standardized care. Examples of such leveraging included commissioning interdisciplinary trauma councils to implement Guideline-concordant protocols and conducting frequent review. Our findings corroborate the methods advocated by other researchers to implement clinical practice guidelines in critical care environments. These methods include guidelines-based clinical management protocols with pre-printed orders, ${ }^{15}$ daily rounds checklists with mandated verbal team review at the point of care, ${ }^{27}$ and frequent adherence reports submitted to a multidisciplinary trauma committee. ${ }^{16,27-31}$

We recommend writing the Guidelines into local protocols to increase institutional and provider focus on the Guidelines and in turn increase adherence. Providers favored and reported stricter adherence to protocols that were developed and implemented locally using consensus models involving all relevant services. Numerous studies-including those in severe adult $\mathrm{TBI}^{32-34}$ successfully used multidisciplinary task forces to design clinical pathways. ${ }^{15,16,29,34-37}$ They included all services that would be impacted, including resistant parties in the non-hierarchical protocol development to elicit clear expectations, ownership, and commitment to its success and compliance through written 
endorsement. ${ }^{34,35,37-39}$ Ongoing protocol assessment and consensus should be facilitated through periodic literature and case review to consider adjustments and trends in adherence and patient outcomes. ${ }^{35,36}$ Trauma centers need to formally initiate development of these councils and commit to implement its decisions by providing administrative support, changes to documentation that reflect protocols/guidelines, active dissemination, and tailored continuing education. ${ }^{15,16,29,37}$ Hospitals should invest in systems to collect baseline and post-protocol data for committee auditing and feedback. ${ }^{15,16,28,29,37,40}$

Providers described varying dynamics between services that share clinical responsibility of pediatric TBI patients. Respectful communication and interdisciplinary cooperation between providers facilitated adherent treatment decisions; the absence of shared expectations resulted in interdisciplinary conflict. Our findings validate the importance of a culture of cooperation characterized by interdisciplinary leadership, collaborative decision making, open communication, and non-hierarchical intensive care unit team dynamics for implementation and adherence to Guidelines in critical care. ${ }^{15,16,35,39}$ For example, our previous work highlighted the importance of timely placement of ICP monitors ${ }^{3}$; in the current study, successful care coordination was explicitly linked to swift placement of ICP monitors. This is vital in severe pediatric TBI care where adherence is dependent upon the actions of multiple units, disciplines, and services. ${ }^{35}$

Severe pediatric TBI care teams need to develop "rules of engagement" for interdisciplinary treatment planning, coordination, and execution. Applicable models from emergency medicine include a planning process that encompasses shared identification, interpretation, and prioritization of team goals and resources, with the development of back-up plans. ${ }^{41,42}$ Coordination covers sequencing of procedures and includes monitoring progress of team goals using multiple methods, such as observation and structured feedback loops. ${ }^{41,42}$ Attention should be given to communicating changes in patient status to co-managing departments. Teams need shared decision-making models for routine and unexpected situations, with closed loop communication to counteract interpretive bias across specialties. ${ }^{37,41}$ These actions need to be supported by strong leadership. ${ }^{41}$

We did not find the commonly reported financial, reimbursement, equipment, diagnostic, or referral constraints, or physician reluctance to work in trauma because of personal and financial inconvenience that has been reported in other studies. ${ }^{35}$ This is likely due to the characteristics of Level 1 trauma centers in general as they differ from primary and secondary care facilities. ${ }^{35}$ The urban and regional university-affiliated pediatric trauma centers we sampled and the providers that elect to staff them expect to treat high volumes of trauma, with relatively poor reimbursement.

There are some study limitations. Results of this study represent a small number of pediatric trauma centers. However, the total number of participants was large. Although moderators attempted to ensure representative discussion time, providers with strong opinions regarding barriers and facilitators to Pediatric TBI Guideline adherence may have been more willing to participate in the focus groups. Study data are based on provider recall and disclosure of events taking place at their worksite in front of professional peers, leading to possible recall or social desirability bias.

\section{Conclusion}

The remediable barriers to Guideline adherence are not physical or financial constraints, but rather the human factors, orga- nizational cultures, and institutional structures that perpetuate silos or fail to actively promote interdisciplinary discussion of evidence-based care. The facilitators include transparent, high functioning teams of providers and the institutional policies that allow them to flourish. Creating a culture of collaboration, delivering standardized pediatric TBI care, and open communication while considering local barriers and facilitators between treatment teams and among providers may facilitate Guideline adherence.

\section{Acknowledgments}

We would like to thank the following coordinators who assisted with organization of focus groups at their respective sites: Rachelle Belle, R.N., at University of Pittsburgh's Children's Hospital of Pittsburgh; Sheila Giles, R.N., at the Nationwide Children's Hospital; Nithya Kannan, M.D., at the University of Washington's Harborview Medical Center; Alma Ramirez at the HarborUniversity of California, Los Angeles Medical Center; and Kristi Schmidt, M.D., at the Anne and Robert H. Lurie Children's Hospital. We would also like to thank Harriet Saxe, J.D., for her assistance with data management and preliminary coding. Funding for this study was provided by National Institute of Neurological Disorders and Stroke grant R01 NS072308-03 (Vavilala) and National Center for Advancing Translational Sciences grant KL2TR000421 (Moore).

\section{Author Disclosure Statement}

No competing financial interests exist.

\section{References}

1. Faul, M., Xu, L., Wald, M., and Coronado, V. (2010). Traumatic Brain Injury in the United States: Emergency Department Visits, Hospitalizations, and Deaths 2002-2006. Centers for Disease Control: Atlanta, GA.

2. Adelson, P., Bratton, S., Carney, N., Chesnut, R., du Coudray, H., Goldstein, B., Kochanek, P., Miller, H., Partington, M., Selden, N., Warden, C., and Wright, D. (2003). Guidelines for the acute medical management of severe traumatic brain injury in infants, children, and adolescents. Pediatr. Crit. Care Med. 4, S1-S75.

3. Kochanek, P., Carney, N., Adelson, P., Ashwal, S., Bell, M., Bratton, S., Carson, S., Chesnut, R., Ghajar, J., Goldstein, B., Grant, G., Kissoon, N., Peterson, K., Selden, N., Tasker, R., Tong, K., Vavilala, M., Wainwright, M., and Warden, C. (2012). Guidelines for the acute medical management of severe traumatic brain injury in infants, children, and adolescents, 2nd edition. Pediatr. Crit. Care Med. 13, S1-S82.

4. Vavilala, M., Kernic, M., Wang, J., Kannan, N., Mink, R., Wainwright, M., Groner, J., Bell, M., Giza, C., Zatzick, D., Ellenbogen, R., Boyle, L., Mitchell, P., and Rivara, F. (2014). Acute care indicators associated with discharge outcomes in children with severe traumatic brain injury. Crit. Care Med. 42, 2258-2266.

5. Jennett, B., Teasdale, G., Braakman, R., Minderhoud, J., and Knilljones, R. (1976) Predicting outcome in individual patients after severe head-injury. Lancet 1, 1031-1034.

6. Kernic, M., Rivara, F., Zatzick, D., Bell, M., Wainwright, M., Groner, J., Giza, C., Mink, R., Ellenbogen, R., Boyle, L., Mitchell, P., Kannan, N., and Vavilala, M. (2013). Triage of children with moderate and severe traumatic brain injury to trauma centers. J. Neurotrauma 30, 1129-1136.

7. Pope, C., Ziebland, S., and Mays, N. (2000). Qualitative research in health care: analysing qualitative data. BMJ. 320, 114-116.

8. Freeman, T. (2006). 'Best practice' in focus group research: making sense of different views. J. Adv. Nurs. 56, 491-497.

9. Graneheim, U., and Lundman, B. (2004). Qualitative content analysis in nursing research: concepts, procedures and measures to achieve trustworthiness. Nurse Educ. Today 24, 105-112. 
10. Hsieh, H., and Shannon, S. (2005). Three approaches to qualitative content analysis. Qual. Health Res. 15, 1277-1288.

11. McPherson, G. and Thorne, S. (2006). Exploiting exceptions to enhance interpretive qualitative health research: insights from a study of cancer communication. Int. J. Qual. Methods 5, 73-86.

12. Trauma Foundation; American Association of Neurological Surgeons; Congress of Neurological Surgeons; Joint Section on Neurotrauma and Critical Care, AANS/CNS. (2007). Guidelines for the management of severe traumatic brain injury, 3rd edition. J. Neurotrauma 24 Suppl, S1-S106.

13. Francl, L.J. (2001). The disease triangle: a plant pathological paradigm revisited. Plant Health Instructor, doi:10.1094/PHI-T- 2001-0517-01.

14. Sinuff, T. and Cook, D. (2003). Guidelines in the intensive care unit. Clin. Chest Med. 24, 739-749.

15. Sinuff, T., Cook, D., Giacomini, M., Heyland, D., and Dodek, P. (2007). Facilitating clinician adherence to guidelines in the intensive care unit: a multicenter, qualitative study. Crit. Care Med. 35, 2083 2089.

16. Cahill, N., Suurdt, J., Ouellette-Kuntz, H., and Heyland, D. K. (2010). Understanding adherence to guidelines in the intensive care unit: development of a comprehensive framework. JPEN J. Parenter. Enteral Nutr. 34, 616-624.

17. Sinuff, T., Eva, K., Meade, M., Dodek, P., Heyland, D., and Cook, D. (2007). Clinical practice guidelines in the intensive care unit: a survey of Canadian clinicians' attitudes. Can. J. Anaesth. 54, 728-736.

18. Cabana, M., Rand, C., Powe, N., Wu, A., Wilson, M., Abboud, P., and Rubin, H. (1999). Why don't physicians follow clinical practice guidelines? A framework for improvement. JAMA 282, 1458-1465.

19. Goossens, A., Bossuyt, P., and de Haan, B. (2008). Physicians and nurses focus on different aspects of guidelines when deciding whether to adopt them: an application of conjoint analysis. Med. Dec. Making $28,138-145$

20. Abrahamson, K., Fox, R., and Doebbeling, B. (2012). Facilitators and barriers to clinical practice guideline use among nurses. Am. J. Nurs. $112,26-35$

21. Janssen, M., Van Achterberg, T., Adriaansen, M., Kampshoff, C., Schalk, D., and Mintjes-de Groot, J. (2012). Factors influencing the implementation of the guideline triage in emergency departments: a qualitative study. J Clin. Nurs. 21, 437-447.

22. Swennen, M., Van der Heijden, G., Boeije, H., Van Rheenen, N., Verheul, F., Van der Graaf, Y., and Kalkman, C. (2013). Doctors' perceptions and use of evidence-based medicine: a systematic review and thematic synthesis of qualitative studies. Acad. Med. 88, 1384-1396.

23. Hymel, K., Makoroff, K., Laskey, A., Conaway, M., and Blackman, J. (2007). Mechanisms, clinical presentations, injuries, and outcomes from inflicted verses noninflicted head trauma during infancy: results of a prospective, multicentered, comparitive study. Pediatrics 119, 922-929.

24. Keenan, H., Runyan, D., Marshall, S., Nocera, M., and Merten, D. (2004). A population-based comparison of clinical and outcome characteristics of younger children with serious inflicted and noninflicted traumatic brain injury. Pediatrics 114, 633-639.

25. Ewing-Cobbs, L., Kramer, L., Prasad, M., Canales, D., Louis, P., Fletcher, J., Vollero, H., Landry, S., and Cheung, K. (1998). Neuroimaging, physical, and developmental findings after inflicted and noninflicted traumatic brain injury in young children. Pediatrics 102 , 300-307.

26. Deans, K., Minneci, P., Lowell, W., and Groner, J. (2013). Increased morbidity and mortality of traumatic brain injury in victims of nonaccidental trauma. J. Trauma Acute Care Surg. 75, 157-160.

27. Byrnes, M., Schuerer, D., Schallom, M., Sona, C., Mazuski, J., Taylor, B., McKenzie, W., Thomas, J., Emerson, J., Nemeth, J., Bailey, R., Boyle, W., Buchman, T., and Coopersmith, C. (2009). Implementation of a mandatory checklist of protocols and objectives improves compliance with a wide range of evidence-based intensive care unit practices. Crit. Care Med. 37, 2775-2781.

28. Teixeira, P., Inaba, K., DuBose, J., Melo, N., Bass, M., Belzberg, H., and Demetriades, D. (2013). Measurable outcomes of quality improvement using a daily quality rounds checklist: two-year prospec- tive analysis of sustainability in a surgical intensive care unit. J. Trauma Acute Care Surg. 75, 717-721.

29. DuBose, J., Inaba, K., Shiflett, A., Trankiem, C., Teixeira, P., Salim, A., Rhee, P., Demetriades, D., and Belzberg, H. (2008). Measurable outcomes of quality improvement in the trauma intensive care unit: the impact of a daily quality rounding checklist. J. Trauma 64, 22-29.

30. Hesdorffer, D. and Ghajar, J. (2007). Marked improvement in adherence to traumatic brain injury guidelines in United States trauma centers. J. Trauma 63, 841-847.

31. English, S., Turgeon, A., Owen, E., Doucette, S., Pagliarello, G., and McIntyre, L. (2013). Protocol management of severe traumatic brain injury in intensive care units: a systematic review. Neurocrit. Care 18 , $131-142$.

32. McIlvoy, L., Spain, D., Raque, G., Vitaz, T., Boaz, P., and Meyer, K (2001). Successful incorporation of the severe head injury guidelines into a phased-outcome clinical pathway. J. Neurosci. Nurs. 33, $72-$ $78,82$.

33. Arabi, Y., Haddad, S., Tamim, H., Al-Dawood, A., Al-Qahtani, S., Ferayan, A., Al-Abdulmughni, I., Al-Oweis, J., and Rugaan, A. (2010). Mortality reduction after implementing a clinical practice guidelines-based management protocol for severe traumatic brain injury. J Crit. Care 25, 190-195.

34. Fakhry, S.M., Trask, A.L., Waller, M.A., and Watts, D.D.; IRTC Neurotrauma Task Force. (2004). Management of brain-injured patients by an evidence-based medicine protocol improves outcomes and decreases hospital charges. J. Trauma 56, 492-499.

35. March, A. (2006). Facilitating Implementation of Evidence-Based Guidelines in Hospital Settings: Learning from Trauma Centers. Commonwealth Fund: New York.

36. LeBlanc, J., Kane-Gill, S., Pohlman, A., and Herr, D. (2012). Multiprofessional survey of protocol use in the intensive care unit. J. Crit. Care 27, 738.e9-738.e17.

37. McCabe, P., and Kalpin, P. (2005). Bold voices in progressive care: using shared decision making to implement evidence-based practice in progressive care. Crit. Care Nurse 25, 76-78, 80-82, 84-87.

38. Institute for Safe Medication Practices. (2010). ISMP Guidelines for Standard Order Sets. Institute for Safe Medication Practices website. Available at: http://ismp.org/tools/guidelines/StandardOrderSets.asp. Accessed June 18, 2014.

39. Curtis, J., Cook, D., Wall, R., Angus, D., Bion, J., Kacmarek, R., Kane-Gill, S., Kirchhoff, K., Levy, M., Mitchell, P., Moreno, R., Pronovost, P., and Puntillo, K. (2006). Intensive care unit quality improvement: a "how-to" guide for the interdisciplinary team. Crit. Care Med. 34, 211-218.

40. Jones, A., Shapiro, N., and Roshon, M. (2007). Implementing early goal-directed therapy in the emergency setting: the challenges and experiences of translating research innovations into clinical reality in academic and community settings. Acad. Emerg. Med. 14, 10721078.

41. Fernandez, R., Kozlowski, S., Shapiro, M., and Salas, E. (2008). Toward a definition of teamwork in emergency medicine. Acad. Emerg. Med. 15, 1104-1112.

42. Fernandez, R., Vozenilek, J., Hegarty, C., Motola, I., Reznek, M., Phrampus, P., and Kozlowski, S. (2008). Developing expert medical teams: toward an evidence-based approach. Acad. Emerg. Med. 15, 1025-1036.

Address correspondence to:

Monica S. Vavilala, MD

University of Washington

Harborview Medical Center

325 Ninth Avenue, Box 359724

Seattle, WA 98104

E-mail: vavilala@u.washington.edu 